Artículo

\title{
Nanopartículas de selenio absorbidas en hidrogeles de quitosán-polivinil alcohol en la producción de pepino injertado
}

\author{
Eduardo Alfonso Treviño López ${ }^{1}$ \\ Alberto Sandoval-Rangel ${ }^{1}$ \\ Adalberto Benavides Mendoza ${ }^{1}$ \\ Hortensia Ortega Ortiz ${ }^{2}$ \\ Gregorio Cadenas Pliego ${ }^{2}$ \\ Marcelino Cabrera de la Fuente ${ }^{1 \S}$ \\ ${ }^{1}$ Departamento de Horticultura-Universidad Autónoma Agraria Antonio Narro. Calzada Antonio Narro \\ núm. 1923, Saltillo, Coahuila, México. CP. 25315. Tel. 8444110333. (eduardo.trevino@ciqa.edu.mx; \\ asandovalr16@gmail.com; abenmen@gmail.com). ${ }^{2}$ Centro de Investigación en Química Aplicada. Blvd \\ Enrique Reyna Hermosillo núm. 140, Saltillo, Coahuila, México. CP. 25294. \\ (hortensia.ortega@ciqa.edu.mx; gregorio.cadenas@ciqa.edu.mx).

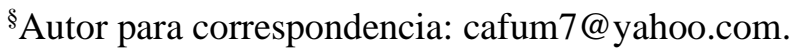

\section{Resumen}

En la búsqueda por mejorar el uso de los recursos naturales y aumentar el rendimiento y la calidad de los frutos empleando técnicas amigables con el medio ambiente, como el injerto y el uso de nanopartículas metálicas. El objetivo de este trabajo fue determinar el efecto del injerto combinado con la aplicación de diferentes concentraciones de nanopartículas de selenio (Se NPs) encapsuladas en un gramo del hidrogel de quitosán-polivinil alcohol (CS-PVA) en la productividad y producción de pepino injertado. Los tratamientos fueron aplicados al sustrato al momento del trasplante de la siguiente manera: 1, 2.5 y $5 \mathrm{mg}$ de NPs de Se absorbidas en un gamo de hidrogeles de CS-PVA, CS-PVA sin NPs y un tratamiento control, en plantas de pepino con injerto y sin injerto. Las variables evaluadas fueron longitud y diámetro de tallo, peso fresco y seco de hojas, área foliar, longitud de raíz, longitud de fruto, número de frutos y rendimiento por planta. Los resultados mostraron que la aplicación de las NPs de Se tienen un efecto benéfico para las plantas, como un agente promotor del crecimiento y que junto con el injerto coadyuvan a obtener una mayor altura de la planta y peso de fruto obteniendo un mayor rendimiento por planta.

Palabras clave: Cucumis sativus, injertos, nanotecnología, rendimiento.

Recibido: enero de 2021

Aceptado: marzo de 2021 


\section{Introducción}

La agricultura moderna requiere prácticas alternativas para mejorar la productividad y calidad de los cultivos, sin afectar el medio ambiente ya que recursos como agua, suelo son cada vez más escasos y la población en aumento. Es por ello investigar alternativas que mejoren la producción agrícola. El injerto en hortalizas permite al cultivo desarrollarse adecuadamente y disminuir el uso de agroquímicos (González et al., 2008), además de tolerar suelos salinos y altas temperaturas (Khah et al., 2006), mejora el rendimiento y calidad de cultivos (Yassin y Hussen, 2015).

Además del uso del injerto en los sistemas agrícolas se están evaluando el uso de nanopartículas (NPs), materiales una dimensión inferior a $100 \mathrm{~nm}$. Este tamaño pequeño da lugar a propiedades diferentes en comparación a escalas mayores, como son alta conductividad, reactividad química, buena relación superficie-volumen (Nel et al., 2006) los que las hace muy útiles en la nutrición de cultivos (Hernández et al., 2018) y la protección de cultivos (Cumplido et al., 2019) a las que muestran los materiales de tamaño micro de la misma composición (Bell et al., 2014). Los principales nanomateriales que se están evaluando en plantas son a base de metales y óxidos como el $\mathrm{Cu}, \mathrm{Fe}, \mathrm{Ce}, \mathrm{Ti}$ y Ag.

Las respuestas celulares son muy diferentes cuando son inducidas por formas iónicas en comparación con las formas nanométricas (Zuverza et al., 2016). Estas nuevas propiedades proporcionan al material un valor agregado que tiene múltiples aplicaciones en la industria agrícola, como los nano-fertilizantes, tratamiento de enfermedades y promoción del crecimiento de las plantas, entre otras (Siddiqui et al., 2015).

El Selenio (Se) es un elemento esencial de la dieta humana (Hartikainen et al., 2005), es necesario para el funcionamiento adecuado del sistema inmunológico tiene funciones estructurales y enzimáticas, por lo cual, es necesario suplementar en suelos o sustratos con deficiencia de selenio, la baja ingesta de selenio puede resultar en varios trastornos de salud, incluyendo enfermedades del corazón, disminución de la fertilidad, el hipotiroidismo, mejora las condiciones relacionadas con el estrés oxidativo. El Se proporciona beneficios a nivel del metabolismo redox, aumentando la resistencia de las plantas a varios factores de estrés biótico y abiótico (Wallace et al., 2009), aumenta el crecimiento y calidad de los frutos (Hernández-Hernández et al., 2019).

Dado que las dosis de Se son pequeñas se requiere el uso de biopolímeros como el quitosán y el CS, un derivado de la quitina encontrado en caparazones de crustáceos, moluscos e insectos (Nge et al., 2006). El CS es capaz de formar hidrogeles cuando se combina con el polivinil alcohol (PVA), un polímero hidrofilico, no toxico, biocomatible, con buena propiedad mecánica y muy estable durante largos periodos de tiempo y en diferente condición de temperatura y $\mathrm{pH}$ (Gholap et $a l .$, 2004) que son capaces de encapsular los ingredientes activos con el fin de mejorar su absorción por las plantas, esta técnica que puede ser eficaz para la biofortificación de cultivos con Se.

México es considerado uno de los principales productores de pepino (Cucumis sativus), ocupa el octavo lugar mundial con una producción de 886270 t en 16206 ha (SIAP 2018). El pepino es una cucurbitácea de gran importancia para consumo en fresco, industria de encurtido e industria cosmética. Una de las grades problemáticas en la producción de esta hortaliza es la presencia de patógenos del suelo, como nematodos y hongos vasculares y debido a esto se han creado métodos 
alternativos en los sistemas de producción, como el uso injerto el cual mejora el crecimiento y la productividad de las plantas en condiciones desfavorables del suelo y del medio ambiente (Rouphael et al., 2012). Para coadyuvar con pa producción del cultivo el objetivo de esta investigación fue determinar el efecto de las NPs de Se sobre la producción y productividad en plantas de pepino con y sin injerto.

\section{Materiales y métodos}

\section{Síntesis de nanopartículas de selenio}

La síntesis de las NPs de Se, se llevó a cabo en el Laboratorio de Síntesis del Centro de Investigación en Química Aplicada (CIQA), en un reactor de vidrio provisto de agitación mecánica, control de temperatura y sistema de atmósfera inerte en medio acuoso empleando ácido selenioso $\left(\mathrm{H}_{2} \mathrm{SeO}_{3}\right)$ y una solución de CS con PVA. Los componentes se mantienen a $0{ }^{\circ} \mathrm{C}$ y la reducción se lleva a cabo con hidracina. Las NPs de Se obtenidas presentan forma esférica y tamaño de partícula de 2 a 20 nm determinados por microscopía de transmisión electrónica (TEM) (Figura 1).

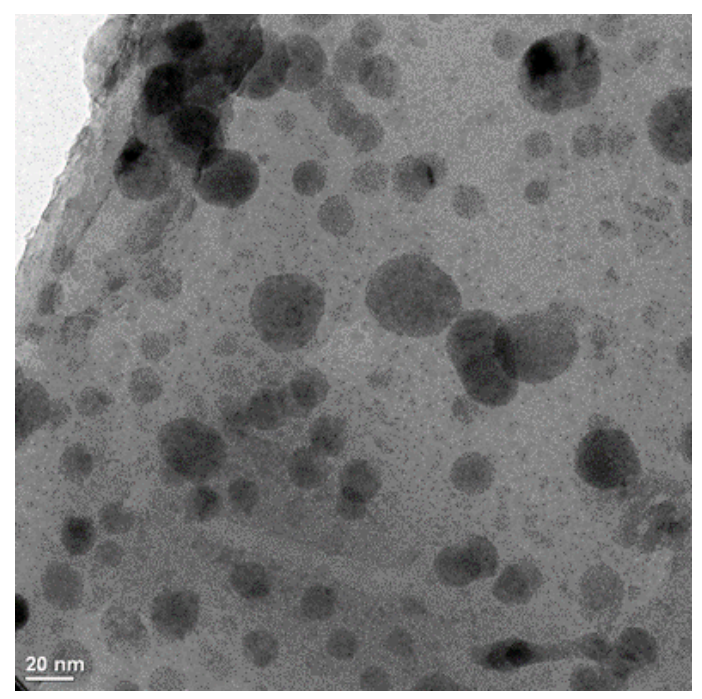

Figura 1. Micrografía de NPs de Se por microscopia de transmisión electrónica desde $\mathbf{2} \mathbf{n m}$ hasta 20 nm, de forma principalmente esféricas.

\section{Absorción de NPs de Se en hidrogeles de CS-PVA}

En una dispersión de NPs de Se, se mezcla con los hidrogeles de CS-PVA para obtener nanocompuestos con 1, 2.5 y $5 \mathrm{mg}$ de Se en un gramo de hidrogeles de CS-PVA secándolos luego a vacío a $60{ }^{\circ} \mathrm{C}$ (Gómez et al., 2017).

\section{Desarrollo experimental del cultivo de pepino}

El desarrollo de la fase agronómica se realizó en las instalaciones de la Universidad Autónoma Agraria Antonio Narro (UAAAN), en el norte de México, cuya ubicación geográfica se encuentra a $25^{\circ} 21^{\prime} 22.51^{\prime}$ ' latitud norte y $101^{\circ} 2$ ' $9.88^{\prime}$ ' longitud oeste y una altitud de $1760 \mathrm{~m}$, durante los 
meses de junio a septiembre de 2017 en un invernadero tipo macrotúnel con cubierta plástica difusa, temperatura promedio diurna de $27{ }^{\circ} \mathrm{C}$ y nocturna de $20{ }^{\circ} \mathrm{C}$, humedad relativa promedio de $60 \%$ y una densidad de plantación de 3 plantas $\mathrm{m}^{-2}$.

\section{Material vegetativo}

La variedad de pepino (Cucumis sativus L.) utilizada fue $c v$ 'Induran' y como portainjerto el híbrido 'cucúrbita Maxima x cucúrbita Moschata' ambos de Rijk Zwaan, The Netherlands.

\section{Siembra}

La variedad de pepino se sembró el 7 de junio de 2017 y el portainjerto el 12 junio de 2017 en charolas de poliestireno de 60 cavidades. El injerto se realizó por el método de aproximación (González et al., 2008) el 22 de junio cuando la variedad y el patrón presentaban diámetros de 4 a $5 \mathrm{~mm}$. Las plántulas ya injertadas se mantuvieron durante tres días en una cámara de prendimiento a una temperatura de $25{ }^{\circ} \mathrm{C}$ y humedad relativa en $80 \%$, antes del trasplante se preparó una mezcla de peat moss y perlita $(1: 1, \mathrm{v} / \mathrm{v})$ la cual fue utilizada como sustrato de crecimiento puesto en bolsas de polietileno negras de $10 \mathrm{~L}$ de capacidad.

Los tratamientos con hidrogeles de CS-PVA se hicieron colocando en la parte baja, media y alta de la maceta para mejor distribución de las NPs de Se en el sustrato y el área de la raíz de la planta, quedando un total de 10 tratamientos con 10 repeticiones (Cuadro 1). Se usó un sistema de riego por goteo para fertilizar con la solución nutritiva de Steiner (Steiner, 1961). La solución nutritiva fue suplementada con diferentes concentraciones de acuerdo con la etapa fenológica del cultivo y de la siguiente manera: $25 \%$ durante el crecimiento, $50 \%$ en la floración, $75 \%$ en el amarre y cuajado del primer fruto y al 100\% durante el llenado de frutos. La conductividad eléctrica de la solución Steiner se mantuvo en $3 \mathrm{mS} \mathrm{cm}^{-1}$.

Cuadro 1. Descripción de los tratamientos estudiados en base a la cantidad de NPs de Se en un gramo de hidrogeles de CS-PVA en macetas de $10 \mathrm{~L}$.

\begin{tabular}{cc}
\hline Tratamiento & Descripción \\
\hline Sin injerto & Sin aplicación \\
& 1 g de CS-PVA \\
& $1 \mathrm{mg}$ de NPs de Se en $1 \mathrm{~g}$ de CS-PVA \\
& $2.5 \mathrm{mg}$ de NPs de Se en $1 \mathrm{~g}$ de CS-PVA \\
Con injerto & $5 \mathrm{mg}$ de NPs de Se en $1 \mathrm{~g}$ de CS-PVA \\
& Sin aplicación \\
& $1 \mathrm{~g}$ CS-PVA \\
& $1 \mathrm{mg}$ de NPs de Se en $1 \mathrm{~g}$ de CS-PVA \\
& $2.5 \mathrm{mg}$ de NPs de Se en $1 \mathrm{~g}$ de CS-PVA \\
$5 \mathrm{mg}$ de NPs de Se en $1 \mathrm{~g}$ de CS-PVA
\end{tabular}




\section{Variables evaluadas}

Las variables evaluadas se hicieron durante el crecimiento (longitud y diámetro de tallo, área foliar, área foliar específica, peso fresco y peso seco de hojas, longitud de raíz, peso fresco de raíz) y al final del ciclo a los 60 días después del trasplante y en la producción (longitud del fruto, diámetro del fruto, peso del fruto, número de frutos, rendimiento por planta) haciendo 16 cortes por planta, cuando el fruto estaba bien formado de color verde oscuro y antes de presentar madurez fisiológica.

\section{Análisis estadístico}

Se empleó un diseño experimental completamente al azar con arreglo factorial (2X5) donde el factor 1 es (con y sin injerto) factor 2 (aplicación de NPs de Se en dosis de 0, 1, 2.5 y 5 mg en un gramo de hidrogeles de CS-PVA e hidrogel de CS-PVA sólo), siendo un total de 10 tratamientos y 10 repeticiones de cada uno. Los resultados se analizaron mediante un análisis de varianza y una prueba de comparación de medias usando la metodología de LSD Fisher con un nivel de significancia de $\leq 0.05$ en el programa InfoStat 2017.

\section{Resultados y discusión}

Los resultados de la evaluación de las variables agronómicas del pepino con y sin injerto son mostrados en el Cuadro 2. se observaron diferencias significativas (LSD, $p \leq 0.05$ ) en longitud del tallo (LT), diámetro de tallo (DT), área foliar área foliar específica (AFE), peso fresco de la hoja $(\mathrm{PFH})$ peso seco de la hoja (PSH) y peso seco fresco de la raíz (PFR).

\section{Efecto del injerto en el desarrollo del cultivo}

Se presenta el efecto del injerto en pepino el cual mostro diferencias significativas donde impacta a la planta positivamente en diferentes etapas del desarrollo. Debido a que tienen una mayor capacidad de obtener nutrientes y agua del suelo, por otra parte, se observó una disminución en el diámetro de tallo en 18\%, menor área foliar, disminución en el peso fresco de la hoja en comparación con plantas no injertadas esto podría ser por el vigor conferido por parte del patrón o porta injerto (Aloni et al., 2010).

Además, se encontró que el efecto del injerto influye positivamente en variables de producción con diferencias significativas $(p \leq 0.05)$ como el aumento en el diámetro y peso de fruto en un $3 \% \mathrm{y}$ $8.5 \%$ con relación a las plantas sin injerto, asimismo para la longitud de fruto, número de frutos y rendimiento por planta los valores más altos fueron obtenidos en los frutos provenientes de plantas injertadas (Colla et al., 2006).

\section{Efecto de las NPs de Se en las variables de crecimiento}

El efecto de la aplicación de las diferentes impacto tanto a plantas injertadas y sin injertar, siendo la dosis de $1 \mathrm{mg}$ de NPs de Se con hidrogeles de CS-PVA sobre las variables de crecimiento como el área foliar y el área foliar específica presentaron diferencias positivas incrementándose la superficie foliar de las plantas en un rango de $10.5 \%$ y $29.6 \%$ respectivamente en relación a los tratamientos sin aplicación de las NPs de Se, este resultado representa una superficie con mayor 
área de intercambio de gases $\left(\mathrm{CO}_{2}\right)$ entre la planta y el medio aéreo, esta variable, se asocia con un aumento de la tasa fotosintética (Ghasemi et al., 2016). El peso fresco de raíz se incrementó $22.4 \%$ con la dosis de $2.5 \mathrm{mg}$ de las NPs de Se con relación a las plantas sin aplicación $(p \leq 0.05)$.

El resto de las variables no presentaron diferencias estadísticas entre las diferentes dosis de aplicación de las NPs de Se. Respecto a las variables de producción el efecto de la aplicación de las NPs de Se solo presentaron diferencias estadísticas $(p \leq 0.05)$ en la variable de número de frutos y el rendimiento por planta en un 26 y $34.9 \%$ respectivamente con relación a las plantas sin aplicación. Asimismo, la longitud, diámetro y peso de fruto, presentaron los valores más altos a esta concentración, aunque sin diferencias significativas. Los resultados del Cuadro 2 se podrían utilizar para suplementar las Nps de Se para elevar la nutrición de las plantas en invernadero (sustrato) y en campo para aumentar la fertilidad del suelo, además las NPs de Se cómo formas de selenio inorgánico pueden influir positivamente en el enraizamiento, crecimiento y productividad en la mayoría de los cultivos hortícolas (Aslani et al., 2014).

Cuadro 2. Efecto de la interacción injerto, NPs de Se y los hidrogeles de CS-PVA en el crecimiento de plantas de pepino.

\begin{tabular}{|c|c|c|c|c|c|c|c|c|}
\hline Injerto & $\begin{array}{l}\text { NPs } \mathrm{Se}+1 \mathrm{~g} \\
\text { CS-PVA }\end{array}$ & $\begin{array}{l}\mathrm{LT} \\
(\mathrm{m})\end{array}$ & $\begin{array}{l}\text { DT } \\
(\mathrm{mm})\end{array}$ & $\begin{array}{l}\mathrm{AF} \\
\left(\mathrm{m}^{2}\right)\end{array}$ & $\begin{array}{c}\mathrm{AFE} \\
\left(\mathrm{cm}^{2} \mathrm{~g}^{-1}\right)\end{array}$ & $\begin{array}{l}\text { PFH } \\
(\mathrm{g})\end{array}$ & $\begin{array}{l}\text { PSH } \\
(\mathrm{g})\end{array}$ & $\begin{array}{c}\text { PFR } \\
(\mathrm{g})\end{array}$ \\
\hline \multirow[t]{10}{*}{ Sin } & 0 & 4.42 & 14.4 & 2.14 & $181.02 \pm 13.5 b$ & 416.8 & 162 & $46.4 \pm 15.37 \mathrm{c}$ \\
\hline & & $\pm 0.1 \mathrm{ab}$ & $\pm 1.23 \mathrm{ab}$ & $\pm 0.1 \mathrm{abc}$ & & $\pm 40.1 \mathrm{ab}$ & $\pm 14.5 \mathrm{ab}$ & \\
\hline & CS-PVA & 4.52 & 14.6 & $2.21 \pm 0.2 \mathrm{ab}$ & 214.35 & 371 & 163.4 & $50 \pm 18.76 c$ \\
\hline & & $\pm 0.6 \mathrm{ab}$ & $\pm 1.59 \mathrm{ab}$ & & $\pm 18.6 \mathrm{ab}$ & $\pm 33.2 \mathrm{bc}$ & $\pm 16.8 \mathrm{ab}$ & \\
\hline & $1 \mathrm{mg}$ CS- & 4.45 & 14.7 & $2.48 \pm 0.1 \mathrm{a}$ & $268.91 \pm 23.5 \mathrm{a}$ & 433.8 & 188.8 & $44.6 \pm$ \\
\hline & PVA & $\pm 0.1 \mathrm{ab}$ & $\pm 0.84 \mathrm{a}$ & & & $\pm 15.9 \mathrm{a}$ & $\pm 23.5 \mathrm{a}$ & $11.76 \mathrm{c}$ \\
\hline & $2.5 \mathrm{mg} \mathrm{CS}-$ & 4.47 & 13.8 & 1.97 & $192.79 \pm 11.3 b$ & 406 & 141.6 & $83.6 \pm 33.66 \mathrm{a}$ \\
\hline & PVA & $\pm 0.1 \mathrm{ab}$ & $\pm 1.09 \mathrm{ab}$ & $\pm 0.05 \mathrm{abc}$ & & $\pm 16.5 \mathrm{ab}$ & $\pm 19.6 \mathrm{bc}$ & \\
\hline & $5 \mathrm{mg} \mathrm{CS}-$ & 4.52 & 13.4 & $2 \pm 0.2 \mathrm{abc}$ & $191.86 \pm 23 b$ & 405.4 & $149 \pm 17.3 \mathrm{a}$ & 51.8 \\
\hline & PVA & $\pm 0.2 \mathrm{ab}$ & $\pm 0.97 \mathrm{bc}$ & & & $\pm 18.3 \mathrm{ab}$ & bc & $\pm 12.35 \mathrm{bc}$ \\
\hline \multirow[t]{10}{*}{ Con } & 0 & 4.44 & 12.4 & 1.93 & $187.54 \pm 15.6 b$ & 344 & 135.4 & $80 \pm 31.2 \mathrm{ab}$ \\
\hline & & $\pm 0.1 \mathrm{ab}$ & $\pm 0.43 \mathrm{~cd}$ & $\pm 0.3 \mathrm{abc}$ & & $\pm 31.2 \mathrm{~cd}$ & $\pm 25.9 \mathrm{bc}$ & \\
\hline & CS-PVA & 4.09 & 11 & $1.86 \pm 0.2 b c$ & $170.33 \pm 13.7 b$ & 313.6 & 136.4 & 59.6 \\
\hline & & $\pm 0.8 \mathrm{~b}$ & $\pm 1.65 \mathrm{de}$ & & & $\pm 36.5 d$ & $\pm 26.3 \mathrm{bc}$ & $\pm 19.8 \mathrm{abc}$ \\
\hline & $1 \mathrm{mg} \mathrm{CS}-$ & 4.15 & $9.6 \pm 0.42 f$ & 2.15 & 209.04 & 305.2 & 125.6 & 58.8 \\
\hline & PVA & $\pm 0.3 \mathrm{~b}$ & & $\pm 0.4 \mathrm{abc}$ & $\pm 17.4 \mathrm{ab}$ & $\pm 45.2 \mathrm{~d}$ & $\pm 38 \mathrm{bc}$ & $\pm 19.4 \mathrm{abc}$ \\
\hline & $2.5 \mathrm{mg} \mathrm{CS}-$ & 4.54 & $9.5 \pm 0.63 f$ & $1.96 \pm 0.4 b c$ & $172.71 \pm 12.5 b$ & 316 & 129.4 & 71.2 \\
\hline & PVA & $\pm 0.8 \mathrm{ab}$ & & & & $\pm 39.1 \mathrm{~cd}$ & $\pm 13.6 \mathrm{bc}$ & $\pm 22.76 \mathrm{abc}$ \\
\hline & $5 \mathrm{mg}$ CS- & 4.64 & $10 \pm 0.54 \mathrm{ef}$ & $1.79 \pm 0.3 \mathrm{c}$ & $165.18 \pm 14.6 b$ & 332.2 & 111.6 & 61.6 \\
\hline & PVA & $\pm 0.6 \mathrm{a}$ & & & & $\pm 24.3 \mathrm{~cd}$ & $\pm 14.6 \mathrm{c}$ & $\pm 26.76 \mathrm{abc}$ \\
\hline
\end{tabular}

$\mathrm{LT}=$ longitud de tallo; $\mathrm{DT}=$ diámetro de tallo; $\mathrm{AF}=$ área foliar, $\mathrm{AFE}=$ área foliar específica; $\mathrm{PFH}=$ peso fresco de hojas; $\mathrm{PSH}=$ peso seco de hojas; $\mathrm{LR}=$ longitud de raíz; $\mathrm{PFR}=$ peso fresco de raíz. CS-PVA= hidrogel de quitosán-polivinil alcohol. Valores con diferentes letras son significativamente diferentes (LSD Fisher $\leq 0.05$ ).

\section{Interacción injerto - NPs de Se en las variables de crecimiento y desarrollo del cultivo}

El efecto mayor entre el injerto y la aplicación de las NPs de Se absorbidas en los hidrogeles de CS-PVA se muestran en el Cuadro 3, donde la longitud del tallo en plantas injertadas con la dosis de $5 \mathrm{mg}$ de NPs de Se aumenta en $4.9 \%$ en relación a las plantas sin injerto y sin aplicación de NPs 
de Se, (Gómez et al., 2017), el injerto confiere un vigor indudable a las plantas, tal vez mejorando su capacidad de absorber agua y nutrientes de su entorno, lo que se traduce en una tasa de crecimiento superior.

Cuadro 3. Efecto de la interacción del injerto y la aplicación de las NPs de Se absorbidas en los hidrogeles de CS-PVA en la producción y rendimiento del cultivo de pepino.

\begin{tabular}{ccccccc}
\hline $\begin{array}{c}\text { Tratamiento } \\
\text { injerto }\end{array}$ & $\begin{array}{c}\text { mg NPs Se }+ \\
\text { 1g CS }\end{array}$ & $\begin{array}{c}\text { LF } \\
(\mathrm{cm})\end{array}$ & $\begin{array}{c}\text { DF } \\
(\mathrm{mm})\end{array}$ & $\begin{array}{c}\text { PF } \\
(\mathrm{g})\end{array}$ & NF & $\begin{array}{c}\text { RP } \\
(\mathrm{kg})\end{array}$ \\
\hline Sin & 0 & $20.3 \pm 1 \mathrm{a}$ & $43.56 \pm 1.3 \mathrm{ab}$ & $236.8 \pm 20.5 \mathrm{bc}$ & $10.2 \pm 2.5 \mathrm{ab}$ & $2.4 \pm 0.7 \mathrm{bcd}$ \\
& CS-PVA & $21.3 \pm 1.1 \mathrm{a}$ & $44.93 \pm 0.8 \mathrm{ab}$ & $257.2 \pm 23.3 \mathrm{abc}$ & $10.8 \pm 2.7 \mathrm{ab}$ & $2.8 \pm 0.7 \mathrm{abc}$ \\
& 1 & $20.1 \pm 1.1 \mathrm{a}$ & $43.26 \pm 1.1 \mathrm{~b}$ & $236.6 \pm 34.4 \mathrm{bc}$ & $11.2 \pm 1.8 \mathrm{ab}$ & $2.7 \pm 0.5 \mathrm{abcd}$ \\
& 2.5 & $21.2 \pm 0.4 \mathrm{a}$ & $43.67 \pm 2.3 \mathrm{~b}$ & $225 \pm 13.7 \mathrm{c}$ & $8 \pm 2.8 \mathrm{~b}$ & $1.8 \pm 0.7 \mathrm{~d}$ \\
Con & 5 & $21.2 \pm 0.5 \mathrm{a}$ & $42.42 \pm 2.41 \mathrm{~b}$ & $248.2 \pm 24.07 \mathrm{abc}$ & $11 \pm 2.6 \mathrm{ab}$ & $2.7 \pm 0.2 \mathrm{abc}$ \\
& 0 & $20.2 \pm 1 \mathrm{a}$ & $43.69 \pm 1.2 \mathrm{ab}$ & $257.6 \pm 21.8 \mathrm{abc}$ & $8.2 \pm 3.2 \mathrm{~b}$ & $2.1 \pm 0.2 \mathrm{~cd}$ \\
& CS-PVA & $21 \pm 0.8 \mathrm{a}$ & $43.9 \pm 0.9 \mathrm{ab}$ & $250.6 \pm 36.8 \mathrm{abc}$ & $10 \pm 2.2 \mathrm{~b}$ & $2.5 \pm 0.6 \mathrm{bcd}$ \\
& 1 & $21 \pm 1.6 \mathrm{a}$ & $45.1 \pm 1.9 \mathrm{ab}$ & $265.2 \pm 26.7 \mathrm{ab}$ & $10.8 \pm 2.1 \mathrm{ab}$ & $3 \pm 0.6 \mathrm{ab}$ \\
& 2.5 & $25.8 \pm 1.2 \mathrm{a}$ & $44.6 \pm 0.5 \mathrm{ab}$ & $257.8 \pm 32.5 \mathrm{abc}$ & $11.4 \pm 1.9 \mathrm{a}$ & $2.9 \pm 0.8 \mathrm{ab}$ \\
& 5 & $28.8 \pm 1.2 \mathrm{a}$ & $46.1 \pm 0.6 \mathrm{a}$ & $280.8 \pm 25.4 \mathrm{a}$ & $12.2 \pm 1.8 \mathrm{a}$ & $3.4 \pm 0.8 \mathrm{a}$ \\
\hline
\end{tabular}

$\mathrm{LF}=$ longitud de fruto; $\mathrm{DF}=$ diámetro de fruto; $\mathrm{PF}=$ peso promedio de fruto; $\mathrm{NF}=$ número de frutos; $\mathrm{RP}=$ rendimiento por planta. Valores con diferentes letras son significativamente diferentes (LSD Fisher $\leq 0.05$ ).

Asimismo (Nawaz et al., 2017) el injerto promueven el crecimiento de brotes y hojas, además que confirma que el Se es un elemento benéfico para las plantas, como un agente promotor del crecimiento (Garcia-Bañuelos et al., 2011) que junto con el injerto causan una altura mayor de la planta (Riga, 2015), el área foliar y peso fresco de las hojas de las plantas injertadas fue menor en comparación a las no injertadas esto puede ser debido a que fueron más eficientes en el consumo de agua y nutrientes.

El desarrollo de la longitud de la raíz fue influenciada solo por el injerto en comparación con el tratamiento sin injerto y sin aplicación de las NPs de Se $(p \leq 0.05)$; sin embargo, estadísticamente la longitud de raíz va disminuyendo a medida que la dosis aplicada de NPs de Se aumenta, en dosis de $1 \mathrm{mg}$ en $8.5 \%$, para $2.5 \mathrm{mg}$ en un $10 \%$ y para $5 \mathrm{mg}$ de NPs de Se un $15 \%$, datos similares fueron encontrados en un estudio en frijol por (Aggarwal et al., 2011), donde la dosis menor a 2 ppm de selenato de sodio promovió el crecimiento de raíces y dosis mayores la inhibieron.

Respecto a la interacción injerto y NPs de Se muestran una tendencia a disminuir la longitud de la raíz de la planta de pepino y el injerto a desarrollar masa radicular. Los resultados obtenidos ya que a mayor dosis de NPs de Se afecta el crecimiento de las raíces en las plantas de pepino, pero el injerto promueve el desarrollo de pelos absorbentes lo cual mejora la absorción de nutrientes y desarrollo de la planta injertada (Nawaz et al., 2017).

En este estudio la variable número de frutos se obtuvo un peso promedio mayor en los tratamientos injertados y con dosis de 2.5 y $5 \mathrm{mg}$ de NPs de Se absorbidas en hidrogel de CS-PVA; esto concuerda con estudios de (Cavalu et al., 2017), donde aplicaron NPs de Se y el crecimiento de las 
plántulas de brócoli dependía de la concentración de selenio, también hay reportes de aplicaciones de selenito sódico $\left(\mathrm{Na}_{2} \mathrm{SeO}_{3}\right)$ en dosis de $2,4,6 \mathrm{mg} \mathrm{L}^{-1}$ en pepino, los resultados obtenidos mostraron que aumentó el rendimiento con $2 \mathrm{mg} \mathrm{L}^{-1}$ y disminuyó en la dosis de $6 \mathrm{mg} \mathrm{L}^{-1}$ (Haghighi et al., 2016).

Las plantas de pepino sin injerto con $1 \mathrm{mg}$ de NPs Se absorbidas en hidrogeles de CS-PVA presentan la mayor área foliar dada por el efecto de la aplicación de selenio, factor determinante en la modificación de la fotosíntesis (Hawrylak et al., 2015) esto puede explicar los efectos benéficos reportados en la aplicación de las dosis adecuadas de Se en las plantas mediante la promoción del crecimiento del área foliar y la productividad (Jiang et al., 2015).

Hay evidencia en la literatura de que el Se puede mejorar la productividad de la planta a través de la mejora de la fotosíntesis, ya que este proceso se estimula en las plantas mediante la suplementación óptima (Gilmara et al., 2017), por otro lado el selenio podría provocar síntomas de la toxicidad a dosis más elevadas que influyen en el crecimiento de la hoja y número y tamaño de estomas del pepino, y en la asimilación de concentración interna de $\mathrm{CO}_{2}$ (Haghighi et al., 2016), este efecto en la maquinaria antioxidante sugiere que con las concentraciones apropiadas de Se podría mejorar significativamente la fotosíntesis.

La disminución del área foliar se presentó en el tratamiento con injerto y con aplicación de 5 mg de NPs de Se, debido que las sustancias metabólicas podrían transferirse del patrón a la variedad incluidas las moléculas de señalización que pueden causar efectos fisiológicos como la señalización hormonal que está implicada en la formación de unión de injertos, la comunicación entre el patrónvariedad que afecta, el crecimiento, rendimiento, floración y la calidad de fruto (Aloni et al., 2010).

El injerto también permite un cambio en la morfología de las hojas y muestra disminución de la conductancia estomática en respuesta al estrés por sequía, para mantener la turgencia de la hoja, (Poudyala et al., 2015). Las plantas injertadas pueden mantener ajuste osmótico en las hojas bajo condiciones de estrés hídrico a través de mecanismos de resistencia al estrés según lo informado por (Nilsen et al., 2014). Además de una estrategia de conservación del agua, basada en la combinación de la reducción del crecimiento (reducción del área foliar) y la capacidad de mantener la fotosíntesis neta a potenciales menores de agua.

El área foliar especifica del pepino fue mayor al aplicar $1 \mathrm{mg}$ de NPs de Se y sin injerto, lo cual indica que el Se modificó el programa de desarrollo aumentando la densidad de la hoja en un 38.5\% teniendo hojas más grandes y menos eficientes en comparación con el tratamiento donde se aplican $5 \mathrm{mg}$ de NPs de Se en plantas injertadas donde el rendimiento por planta fue de $21 \%$ mayor que el tratamiento sin injerto y $1 \mathrm{mg}$ de NPs de Se, lo que se traduce en mayor eficiencia en la asimilación de la mayor proporción de radiación, una alta tasa de asimilación de $\mathrm{CO}_{2}$, y translocación de gran cantidad de asimilados hacia los frutos de la planta.

En contraste con los tratamientos que presentaron valores más altos de área foliar, podemos decir que el área foliar (AF) obtenida en el tratamiento con injerto y sin NPs de Se presenta un incremento de $0.96 \%$ y un rendimiento de $38 \%$ respecto al testigo. Las NPs de Se podrían incrementar el área foliar en plantas de pepino sin injerto y provocar una sinergia con el nitrógeno promoviendo crecimiento vegetativo e inhibiendo la promoción de frutos donde la señalización promovería el desarrollo de hojas y aborto de frutos (Hartikainen, 2000). 
Respecto al número de frutos y el rendimiento, la combinación sin injerto y $5 \mathrm{mg}$ de NPs de Se modificaron positivamente estas variables, lo cual puede ser atribuido a que la planta en esta dosis sufriera una fisiopatía por parte de selenio por lo que se podría reaccionar en aborto de los frutos (Kaur et al., 2014).

\section{Conclusiones}

El rendimiento agronómico de las plantas de pepino se incrementa en plantas sin injertar, pero tratadas con la dosis de 1 y $5 \mathrm{mg}$ de NPs de Se, en tanto que la dosis que produjo el mayor rendimiento productivo se presentó cuando a las plantas de pepino injertadas se les aplico la dosis de $5 \mathrm{mg}$ de NPs de Se, lo que afectó positivamente la longitud del fruto, el diámetro del tallo y el peso fresco. Aunque las plantas injertadas en combinación con las NPs de Se tienden a disminuir el área foliar esta combinación dio como resultado una planta más eficiente en cuanto al uso y consumo de agua. El mayor incremento en el crecimiento del área foliar y desarrollo vegetal fue obtenido en plantas de pepino sin injertar y con la aplicación de 1mg de NPs de Se absorbidas en hidrogeles de CS-PVA.

\section{Literatura citada}

Aggarwal, M.; Sharma, S.; Kaur, N. and Pathania, D. 2011. Exogenous proline application reduces phytotoxic effects of selenium by minimising oxidative stress and improves growth in bean (Phaseolus vulgaris L). 140:354-367. doi.org/10.1007/s12011-010-8699-9.

Aloni, B.; Cohen, R.; Karni, L.; Aktas, H. and Edelstein, M. 2010. Hormonal signaling in rootstockscion interactions. Sci. Hortic. 127(2):119-126. doi.org/10.1016/j.scienta.2010.09.003.

Aslani, F.; Bagheri, S.; Muhd-Julkapli, N.; Juraimi, A. S.; Hashemi, F. S. G. and Baghdadi, A. 2014. Effects of engineered nanomaterials on plants growth: an overview. Scientific World J. doi.org/10.1155/2014/641759.

Bell, I. R.; Ives, J. A. and Jonas, W. B. 2014. Nonlinear effects of nanoparticles: biollgical variability from hormetic doses, small particles sizes, and dinamic adaptative interactions. 12(2):202-232. doi.org/10.2203/dose-response.13-025. Bell.

Cavalu, S. 2017. Effect of nano-se particles supply on assimilator pigments, phenols content and antioxidant capacity of broccoli sprouts. Wulfenia J. 24(7):37-46.

Colla, G.; Roupahel, Y.; Cardarelli, M.; Vegetale, P.; Tuscia, U.; De, S. C. and Rea, E. 2006. Effect of salinity on yield, fruit quality, leaf gas exchange, and mineral composition of grafted watermelon plants. 41(3):622-627.

Cumplido-Nájera, C. F.; González-Morales, S. and Ortega-Ortiz, H. 2019. The application of copper nanoparticles and potassium silicate stimulate the tolerance to Clavibacter michiganensis in tomato plants. Sci. Hortic. 245(1):82-89. Doi.org/10.1016/j.scienta. 2018.10.007.

Garcia-Bañuelos, M. L.; Hermosillo-Cereceres, M. A. and Sánchez, E. 2011. The importance of selenium biofortification in food crops. 7(3):181-190.

Ghasemi, Y.; Ghasemi, K.; Pirdashti, H. and Asgharzadeh, R. 2016. Effect of selenium enrichment on the growth, photosynthesis and mineral nutrition of broccoli. 8(2):199-203. doi.org/10.15835/nsb.8.2.9804. 
Gholap, S. G.; Jog, J. P. and Badiger, M. V. 2004. Synthesis and characterization of hydrophobically modified poly (vinyl alcohol) hydrogel membrane. Polymer. 45(17):5863-5873. doi.org/10.1016/j.polymer.2004.06.032.

Gilmara, P. da S.; Leonardo, C. C.; Víctor, V. C.; Sylvia, L. O. S. and Edilaine, I. F. T. 2017. Selenium and agricultural crops. Afr. J. Agric. Res. 12(32):2545-2554. doi.org/10.5897/ AJAR2016.11884.

Gómez, H. G.; Godina, F. R.; Ortiz, H. O.; Mendoza, A. B.; Torres, V. R. and De La Fuente, M. C. 2017. Use of chitosan-PVA hydrogels with copper nanoparticles to improve the growth of grafted watermelon. Molecules. 22(7):1-9. doi.org/10.3390/molecules22071031.

González, F. M.; Hernández, A.; Casanova, A.; Depestre, T.; Gómez, L.; Rodríguez, M. G. and Liliana, H. 2008. El injerto herbáceo: alternativa para el manejo de plagas del suelo. Rev. Protección Veg. 23(2):69-74.

Haghighi, M.; Sheibanirad, A. and Pessarakli, M. 2016. Effects of selenium as a beneficial element on growth and photosynthetic attributes of greenhouse cucumber. J. Plant Nutri. 39(10):1493-1498. doi.org/10.1080/01904167.2015.1109116.

Hartikainen, H.; Xue, T, and Vieno, P; Helinä. 2000. Selenium as an anti-oxidant and pro-oxidant in ryegrass. Plant and Soil. 225(2):193-200. doi.org/10.1023/A.

Hartikainen, H. 2005. Biogeochemistry of selenium and its impact on food chain quality and human health. J. Trace Elements Medicine Biol. 18(4):309-318. doi.org/10.1016/j.jtemb.2005. 02.009 .

Hawrylak-Nowak, B.; Matraszek, R. and Pogorzelec, M. 2015. The dual effects of two inorganic selenium forms on the growth, selected physiological parameters and macronutrients accumulation in cucumber plants. Acta Physiologiae Plantarum. 37(2):1-13. doi.org/10.1007/s11738-015-1788-9.

Hernández-Hernández, H.; Juárez-Maldonado, A.; Benavides-Mendoza, A.; Ortega-Ortiz, H.; Cadenas-Pliego, G.; Sánchez-Aspeytia, D. and González-Morales, S. 2018. Chitosan-PVA and copper nanoparticles improve growth and overexpress the SOD and JA genes in tomato plants under salt stress. Agronomy. 8(9):175-185. doi.org/10.3390/agronomy8090175.

Hernández-Hernández, H.; Quiterio-Gutiérrez, T.; Cadenas-Pliego, G.; Ortega-Ortiz, H.; Hernández-Fuentes, A. D.; De La Fuente, M. C. and Juárez-Maldonado, A. 2019. Impact of selenium and copper nanoparticles on yield, antioxidant system, and fruit quality of tomato plants. Plants. 8(10):1-17. doi.org/10.3390/plants8100355.

Jiang, Y.; Z. H. Z.; Bu, Y.; C. Z.; R.; J. Z. L.; J. J. H. and Y. G. H. 2015. Effects of selenium fertilizer on grain yield, Se uptake and distribution in common buckwheat (Fagopyrum esculentum Moench). Plant, Soil Environ. 61(8):371-377. doi.org/10.17221/284/2015PSE.

Kaur, N.; Sharma, S.; Kaur, S. and Nayyar, H. 2014. Selenium in agriculture: a nutrient or contaminant for crops. Archv. Agron. Soil Sci. 60(12):1593-1624. doi.org/10.1080/ 03650340.2014.918258.

Khah, E. M.; Kakava, E.; Mavromatis, A.; Chachalis, D. and Goulas, C. 2006. Effect of grafting on growth and yield of tomato (Lycopersicon esculentum Mill.) in greenhouse and opentield. J. Appl. Hortic. 08(01):3-7. doi.org/10.37855/jah.2006.v08i01.01.

Nawaz, M. A.; Wang, L.; Jiao, Y.; Chen, C.; Zhao, L.; Mei, M. and Huang, Y. 2017. Pumpkin rootstock improves nitrogen use efficiency of watermelon scion by enhancing nutrient uptake, cytokinin content, and expression of nitrate reductase genes. Plant Growth Regulation. 82(2):233-246. doi.org/10.1007/s10725-017-0254-7. 
Nge, K. L.; Nwe, N.; Chandrkrachang, S. and Stevens, W. F. 2006. Chitosan as a growth stimulator in orchid tissue culture. Plant Sci. 170(6):1185-1190. doi.org/10.1016/j.plantsci.2006. 02.006 .

Nilsen, E. T.; Freeman, J.; Grene, R. and Tokuhisa, J. 2014. A rootstock provides water conservation for a grafted commercial tomato (Solanum lycopersicum L.) line in response to mild-drought conditions: a focus on vegetative growth and photosynthetic parameters. PloS One. 9(12):115380. doi.org/10.1371/journal.pone.0115380.

Poudyala, D.; Khatria, L. and Uptmoora, R. 2015. Advances in crop science and technology an introgression of Solanum habrochaites in the rootstock improves stomatal regulation and leaf area development of grafted tomatoes under drought and low root-zone-temperatures. Adv Crop Sci Tech, 3(3):1-11. doi.org/10.4172/2329-8863.1000175.

Riga, P. 2015. Effect of rootstock on growth, fruit production and quality of tomato plants grown under low temperature and light conditions. Hortic. Environ. Biotechnol. 56(5):626-638. doi.org/10.1007/s13580-015-0042-0.

Rouphael, Y.; Cardarelli, M.; Lucini, L.; Rea, E. and Colla, G. 2012. Nutrient Solution Concentration Affects Growth, Mineral Composition, Phenolic Acids, and Flavonoids in Leaves of Artichoke and Cardoon. HortSci. 47(10):1424-1429. doi.org/10.1007/s11099012-0002-1.

SIAP-SAGARPA. 2018. Servicio de información agroalimentaria y pesquera http//infosiap.gob.mx.

Siddiqui, M. H.; Al-Whaibi, M. H. and Mohammad, F. 2015. Nanotechnology and plant sciences: Nanoparticles and their impact on plants. 77-99 pp. doi.org/10.1007/978-3-319-14502-0.

Steiner, A. A. 1961. A universal method for preparing nutrient solutions of a certain desired composition. Plant and Soil. 15(2):134-154. doi.org/10.1007/BF01347224

Wallace, K.; Kelsey, K. T.; Schned, A.; Morris, J. S.; Andrew, A. S. and Karagas, M. R. 2009. Selenium and risk of bladder cancer: a population-based case-control study. Cancer Prevention Res. 2(1):70-73. doi.org/10.1158/1940-6207.CAPR-08-0046.

Yassin, H. and Hussen, S. 2015. Reiview on role of grafting on yield and quality of selected fruit vegetables. Global J. Sci. Frontier Res. 15(1):1-16.

Zuverza, N.; Martínez, D.; Du, W.; José, A.; Bonilla, N.; López, M. L. and Gardea, J. L. 2016. Exposure of engineered nanomaterials to plants: insights into the physiological and biochemical responses-a review. Plant Physiol. Biochem. doi.org/10.1016/j.plaphy. 2016.05.037. 